\title{
Local Dynamics of an SVIR Epidemic Model with Logistic Growth
}

\author{
Joko Harianto' ${ }^{1}$ Inda Puspita Sari² \\ ${ }^{1}$ Department of Mathematics, Cenderawasih University, \\ ${ }^{2}$ Faculty of Medicine, Cenderawasih University, \\ Email: joharijpr88@gmail.com, indasarira@yahoo.co.id
}

\begin{abstract}
Discussion of local stability analysis of SVIR models in this article included in the scope of applied mathematics. The purpose of this discussion was to provide results of local stability analysis that had not to discuss in some articles related to the SVIR model. The SVIR models discussed in this article involve logistics growth in the vaccinated compartment. The results obtained, i.e., if the basic reproduction number $\mathfrak{R}_{0}<1$ and $m>0$, then there is one equilibrium point i.e. $E^{0}$ is locally asymptotically stable. In the field of epidemiology, this means that the disease will disappear from the population. However, if $\Re_{0}>1, \beta_{1}>\beta$ and $m>0$, then there are two equilibrium points, i.e., disease-free equilibrium point denoted by $E^{0}$ and the endemic equilibrium point denoted by $E_{1}^{*}$. In this case, the endemic equilibrium point $E_{1}^{*}$ Locally asymptotically stable. In the field of epidemiology, this means that the disease will remain in the population. The numerical simulation supports these results.
\end{abstract}

Keywords: Local Dynamics; SVIR Model; Logistic Growth

\section{INTRODUCTION}

The mathematical modeling of infectious disease spread is one of the areas of research for mathematicians and epidemiologists. The formulated model is often said to be a model epidemic. Epidemic models sometimes involve complexity and non-linearity. Therefore, not all epidemic models can found solutions with analytical methods. Epidemic models usually formulate with a deterministic or stochastic approach. Kermack and Mckendrick first introduced a deterministic model in the article [1], which was later generalized by Capasso and Serio in the paper [2]. In the article [2], The population is divided into three parts, i.e., susceptible, infected, and recovered. Each population contains some people whose health conditions correspond to the name of the population. The model is known as the SIR model.

The SIR model represents the dynamic epidemic of a person infected to a susceptible person through direct contact with the closed population. The SIR model formulate as an initial value condition of the ordinary differential equation system, and it is analyzed mathematically. This SIR Model is then continuously developed by many researchers for various cases occurring today. Based on the SIR model developed by Capasso and Serio, the spread of infectious diseases can formulate by dividing the population into some compartment that depends on health conditions. Therefore, the vaccine given to a person can also be considered a specific situation to add to its compartment. People who have given vaccines are included in the $V$ population [3]. The $V$ population is then added to the SI model to study the dynamics of the spread of tuberculosis disease. The SIV model later generalizes into the SVIR model in the article [4], which discusses the possibility that 
individuals can recover from the disease. Furthermore, the SVIR model develops to study the transmission dynamics of influenza disease discussed in articles [5] and [6].

The vaccination strategy discussed in some of the above articles is a continuously given vaccination. Some cases found that vaccines sometimes administer for a certain period or seasonal. Vaccination strategies for this state are usually called Pulse Vaccination Strategy (PVS). A comparison between the continuous vaccination model and the PVS discuss in the article [7]. Later, the model of the continuous vaccination strategy mentioned by Liu et al. was developed by Khan et al., Islam, Harianto, and Suparwati. In the article [8], the non-linear saturation rate adds to the SVIR model with a continuous vaccination strategy. The addition of population migration factors in the susceptible population of the SVIR model with a continuous vaccination strategy discuss in the article [9]. In the paper [10], the SVIR model with a continuous vaccination strategy was developed by adding a death factor due to disease infection. In other words, the infectious disease is a deadly disease. The discussion also provided numerical simulation to support the analysis results. The death rate of each population in the SVIR model is assumed to be identical. A year later, Harianto and Suparwati continued discussing the SVIR model by adding different death rate assumptions for each population in the SVIR model. Its numerical analysis and simulation discuss in the article [11]. In the paper [1] until [11], The population growth assumes to increase exponentially. The models in these articles are more realistic if their population growth uses logistic growth. In other words, the population assumes to be growing in logistics. The logistics growth factor on the SIR model discusses in the articles [12],[13],[14],[15], and [16]. The paper in [17] and [18] also discusses other compartment models that use logistical growth. Li et al. discuss the paper [12] as a SIR model with a saturation incidence rate. According to the article, these discussions will be modified SVIR models in [7] by involving the logistic growth in the population studied in [12]. Furthermore, it discussed the existence of the equilibrium point and local stability. The last part makes a simulated model that has analysis.

\section{METHODS}

This study uses the literature review method. The references used are in the form of several reputable international scientific books and journals. The theories used to support the results of this study are the equilibrium point stability theory of ordinary differential equation systems described in a book written by Perko [19] and the theory related to the construction of the basic reproduction number of an epidemiological model in article [20]. The flow of thought in this discussion, first adds assumptions to the model that has been studied, then forms the model. Next, determine the equilibrium point of the system and analyze its stability. Finally, a numerical simulation is given to check the correctness of the analysis results obtained and a sensitivity analysis of the basic reproduction number.

\section{RESULTS AND DISCUSSION}

\section{Model}

The model discussed in this section is a modification of the SVIR model with a continuous vaccination strategy adopted from the article [7]. The modification of the model is the growth of its population using logistics growth and the death rate caused by disease involved. This model divides the human population into four epidemiological classes, i.e., susceptible (S), infected (I), vaccinated (V), and recovered (R). The dynamic spread of the disease for the case formulate as the following ordinary differential equation system. 


$$
\begin{aligned}
& \frac{d S}{d t}=r S\left(1-\frac{S}{K}\right)-\beta S I-(\mu+\alpha) S \\
& \frac{d V}{d t}=\alpha S-\beta_{1} V I-\left(\mu+\gamma_{1}\right) V \\
& \frac{d I}{d t}=\beta S I+\beta_{1} V I-(\gamma+\mu+\omega) I \\
& \frac{d R}{d t}=\gamma_{1} V+\gamma I-\mu R
\end{aligned}
$$

with the initial conditions $S(0)=S_{0}>0, V(0)=V_{0}>0, I(0)=I_{0}>0$ dan $R(0)=R_{0} \geq$ 0. $r$ denotes the intrinsic growth rate of susceptible population, $K$ is the carrying capacity of the area. $\beta$ is the contact rate between susceptibles and infected, $\mu$ is the natural death rate, $\alpha$ is the rate at which an individual leaves the class of the susceptible by becoming the vaccination process. $\beta_{1}$ is the contact rate between vaccinated and infected, $\gamma_{1}$ is the recovery rate from the vaccinated class, $\gamma$ is the natural recovery rate from the infected class. $\omega$ is the rate of death caused by disease.

\section{Theorem 1}

All solutions of the System (1) with initial conditions $\left(S_{0}, V_{0}, I_{0}, R_{0}\right) \in \mathbb{R}_{+}^{4}$ are positive and bounded for all $t \geq 0$.

\section{Proof:}

The System (1) can be written

$$
\frac{d \tilde{x}}{d t}=\tilde{f}(\tilde{x})
$$

with $\tilde{x}=(S, V, I, R) \in \mathbb{R}^{4}$ and $\tilde{f}(\tilde{x})=\left(f_{1}(\tilde{x}), f_{2}(\tilde{x}), f_{3}(\tilde{x}), f_{4}(\tilde{x})\right) \in \mathbb{R}^{4}$. Clearly $\tilde{f}(\tilde{x})$ differentiable continuous for all $\tilde{x} \in \mathbb{R}^{4}$ such that the System (1) has a unique solution. If we let $G(t)=\beta_{1} I(t)+\mu+\gamma_{1}$, then the second equation of the System (1) can be written

$$
\frac{d V(t)}{d t}+G(t) V(t)=\alpha S(t)
$$

multiplying both sides of the Equation (2) by $\exp \left(\int_{0}^{t} G(u) d u\right)$ gives

$$
\frac{d V(t)}{d t} \exp \left(\int_{0}^{t} G(u) d u\right)+G(t) V(t) \exp \left(\int_{0}^{t} G(u) d u\right)=\alpha S(t) \exp \left(\int_{0}^{t} G(u) d u\right)
$$

By using multiplication rules on derivatives we have

$$
\frac{d}{d t}\left[V(t) \exp \left(\int_{0}^{t} G(u) d u\right)\right]=\alpha S(t) \exp \left(\int_{0}^{t} G(u) d u\right)
$$

By integrating two sides of the Equation (3) with $t$ gives

$$
V(t)=V_{0} \exp \left(-\int_{0}^{t} G(u) d u\right)+\left[\int_{0}^{t} \alpha S(\tau) \exp \left(\int_{0}^{\tau} G(u) d u\right) d \tau\right] \exp \left(-\int_{0}^{t} G(u) d u\right)>0 .
$$

Similarly, we can prove that $S(t), I(t)$ dan $R(t)$ are nonnegative for all $t>0$. Consequently, from the first equation of the System (1), we have

$$
\frac{d S}{d t}=r S\left(1-\frac{S}{K}\right)-\beta S I-(\mu+\alpha) S<r S\left(1-\frac{S}{K}\right)
$$

Hence, there exists $M=\max \left\{S_{0}, K\right\}$ such that $\lim _{t \rightarrow \infty} \sup S(t)<M$

Let $N(t)=S(t)+V(t)+I(t)+R(t)$, total population change is

$\frac{d N}{d t}=\frac{d S}{d t}+\frac{d V}{d t}+\frac{d I}{d t}+\frac{d R}{d t}$ 


$$
=r S\left(1-\frac{S}{K}\right)-\mu N-\omega I<r S-\mu N<r M-\mu N
$$

Then we have $0<N(t)<\frac{r M}{\mu}$ for $t \rightarrow \infty$. Therefore, all solutions of the System (1) are positive and bounded all $t \geq 0$.

\section{Equilibria and Basic Reproduction Number}

We see that $R$ does not appear in three equations in the System (1). Hence, the fourth equation on the System (1) can be ignored, and the discussion is focused on three equations as follows:

$$
\begin{aligned}
& \frac{d S}{d t}=r S\left(1-\frac{S}{K}\right)-\beta S I-(\mu+\alpha) S \\
& \frac{d V}{d t}=\alpha S-\beta_{1} V I-\left(\mu+\gamma_{1}\right) V \\
& \frac{d I}{d t}=\beta S I+\beta_{1} V I-(\gamma+\mu+\omega) I
\end{aligned}
$$

The equilibrium point of the System (4) obtained by resolving the following equations:

$$
\begin{array}{r}
r S\left(1-\frac{S}{K}\right)-\beta S I-(\mu+\alpha) S=0 \\
\alpha S-\beta_{1} V I-\left(\mu+\gamma_{1}\right) V=0 \\
\beta S I+\beta_{1} V I-(\gamma+\mu+\omega) I=0
\end{array}
$$

Clearly from the Equation (3), (4), and (5) that if $m=r-(\mu+\alpha)>0$, then System (4) has a unique disease-free equilibrium point. The disease-free equilibrium point of the System (4) denoted by $E^{0}=\left(S^{0}, V^{0}, I^{0}\right)$ with $S^{0}=\frac{K m}{r}, V^{0}=\frac{K \alpha m}{r\left(\mu+\gamma_{1}\right)}, I^{0}=0$.

The basic reproduction number in epidemiology used to indicate the number of diseases and control spread. The basic reproduction number denoted by $\mathfrak{R}_{0}$. If $\mathfrak{R}_{0}<1$ then the spread of disease in the population predicted to disappear. However, if $\mathfrak{R}_{0}>1$ then the virus will remain in the population. The basic reproduction number of the System (4) in this discussion is determined based on the theory learned in the article [21]. Let $X=$ $(S, V), X^{0}=\left(S^{0}, V^{0}\right), Z=(I), h(X, Z)=\beta S I+\beta_{1} V I-n I$ with $n=\gamma+\mu+\omega>0$ and

Derivating $h\left(X^{0}, Z\right)$ by $Z$ gives

$$
f(X, Z)=\left[\begin{array}{c}
r S\left(1-\frac{S}{K}\right)-\beta S I-(\mu+\alpha) S \\
\alpha S-\beta_{1} V I-\left(\mu+\gamma_{1}\right) V
\end{array}\right]
$$

We have

$$
\left.D_{z} h\left(X^{0}, Z\right)\right|_{z=0}=\beta S^{0}+\beta_{1} V^{0}-n
$$

$$
A=\left.D_{z} h\left(X^{0}, Z\right)\right|_{z=0}=\beta S^{0}+\beta_{1} V^{0}-n
$$

because $A=M-D$ then gives $M=\beta S^{0}+\beta_{1} V^{0}$ and $D=n$. Hence, we have

with $m=r-(\mu+\alpha)$ and $n=\gamma+\mu+\omega$.

$$
\mathfrak{R}_{0}=\rho\left(M D^{-1}\right)=\frac{\beta S^{0}+\beta_{1} V^{0}}{n}=\frac{\beta K m\left(\mu+\gamma_{1}\right)+\beta_{1} K \alpha m}{r n\left(\mu+\gamma_{1}\right)}
$$

The following discussed the existence of the endemic equilibrium point denoted by $E^{*}=\left(S^{*}, V^{*}, I^{*}\right)$. Assume that $S^{*}, I^{*}, V^{*} \neq 0$. From Equation (5), we have

then

$$
r\left(1-\frac{S}{K}\right)-\beta I-(\mu+\alpha)=0
$$


From Equation (6), we have

$$
S^{*}=\frac{K}{r}\left(m-\beta I^{*}\right)
$$

From Equation (7), we have

$$
V^{*}=\frac{\alpha S^{*}}{\beta_{1} I^{*}+\mu+\gamma_{1}}=\frac{K \alpha\left(m-\beta I^{*}\right)}{r\left(\beta_{1} I^{*}+\mu+\gamma_{1}\right)}
$$

$$
\beta S^{*}+\beta_{1} V^{*}-n=0
$$

with $n=\gamma+\mu+\omega$. If $S^{*}=\frac{K}{r}\left(m-\beta I^{*}\right)$ and $V^{*}=\frac{K \alpha\left(m-\beta I^{*}\right)}{r\left(\beta_{1} I^{*}+\mu+\gamma_{1}\right)}$ substituted to Equation (8), then

with

$$
A_{1} I^{* 2}+A_{2} I+A_{3}=0
$$

$A_{1}=\frac{K}{r} \beta^{2} \beta_{1}$

$A_{2}=\frac{K}{r} \beta^{2}\left(\mu+\gamma_{1}\right)+\frac{K}{r} \beta \beta_{1} \alpha+n \beta_{1}-\frac{K}{r} \beta \beta_{1} m$

$A_{3}=n\left(\mu+\gamma_{1}\right)-\frac{K}{r} \beta m\left(\mu+\gamma_{1}\right)-\frac{K}{r} \beta_{1} m \alpha=n\left(\mu+\gamma_{1}\right)\left(1-\mathfrak{R}_{0}\right)$

Hence, $I^{*}$ is the root of the Equation (9), i.e.:

$$
I_{1,2}^{*}=\frac{-A_{2} \pm \sqrt{A_{2}^{2}-4 A_{1} n\left(\mu+\gamma_{1}\right)\left(1-\mathfrak{R}_{0}\right)}}{2 A_{1}}
$$

Clearly that if $\Re_{0}<1$ then $r n>K \beta m$, consequently $A_{2}>0$. Thus, the existence of the endemic equilibrium point depends on $\mathfrak{R}_{0}$. The existence of disease-free and the endemic equilibrium points associated with basic reproductive numbers summarized in the following theorems.

\section{Theorem 2}

We define

$\mathfrak{R}_{0}=\frac{\beta K m\left(\mu+\gamma_{1}\right)+\beta_{1} K \alpha m}{r n\left(\mu+\gamma_{1}\right)}$

$g\left(I^{*}\right)=A_{1} I^{* 2}+A_{2} I+n\left(\mu+\gamma_{1}\right)\left(1-\mathfrak{R}_{0}\right)$

$A_{1}=\frac{K}{r} \beta^{2} \beta_{1}$

$A_{2}=\frac{K}{r} \beta^{2}\left(\mu+\gamma_{1}\right)+\frac{K}{r} \beta \beta_{1} \alpha+n \beta_{1}-\frac{K}{r} \beta \beta_{1} m$

with $m=r-(\mu+\alpha)$ and $n=\gamma+\mu+\omega>0$.

i. $\quad$ If $\Re_{0}<1$ and $m>0$ then $A_{2}>0$ such that there exists a unique equilibrium point of the System (4), i.e. the disease-free equilibrium point denoted by $E^{0}=$ $\left(\frac{K m}{r}, \frac{K \alpha m}{r\left(\mu+\gamma_{1}\right)}, 0\right)$.

ii. If $\mathfrak{R}_{0}>1$ and $m>0$, then System (4) has only two equilibrium point, i.e. the disease-free equilibrium point denoted by $E^{0}=\left(\frac{K m}{r}, \frac{K \alpha m}{r\left(\mu+\gamma_{1}\right)}, 0\right)$ and the endemic equilibrium point denoted by $E_{1}^{*}=\left(S_{1}^{*}, V_{1}^{*}, I_{1}^{*}\right)$ with $S_{1}^{*}=\frac{K}{r}\left(m-\beta I^{*}\right), V_{1}^{*}=$ $\frac{K \alpha\left(m-\beta I^{*}\right)}{r\left(\beta_{1} I^{*}+\mu+\gamma_{1}\right)}$ and $I_{1}^{*}$ is the positive root of $g\left(I^{*}\right)$.

iii. if $\mathfrak{R}_{0}=1, m>0$ and $A_{2}<0$, then System (4) has only two equilibrium point, i.e. the disease-free equilibrium point denoted $E^{0}=\left(\frac{K m}{r}, \frac{K \alpha m}{r\left(\mu+\gamma_{1}\right)}, 0\right)$ and the endemic 
equilibrium point denoted $E_{2}^{*}=\left(S_{2}^{*}, V_{2}^{*}, I_{2}^{*}\right)$ with $S_{2}^{*}=\frac{K}{r}\left(m-\beta I^{*}\right), \quad V_{2}^{*}=$ $\frac{K \alpha\left(m-\beta I^{*}\right)}{r\left(\beta_{1} I^{*}+\mu+\gamma_{1}\right)}$ and $I_{2}^{*}=-\frac{A_{2}}{A_{1}}$.

\section{Local Stability Analysis}

This section discusses the local stability analysis of disease-free equilibrium and endemic points of the System (4). The local stability of the equilibrium point of the System (4) is determined using the Jacobian matrix (Linearization method) studied in [19]. The Jacobian matrix of Systems (4) written as follows:

$$
J=\left[\begin{array}{ccc}
\frac{-2 r S}{K}-\beta I+m & 0 & -\beta S \\
\alpha & -\beta_{1} I-\left(\mu+\gamma_{1}\right) & -\beta_{1} V \\
\beta I & \beta_{1} I & \beta S+\beta_{1} V-n
\end{array}\right]
$$

with $m=r-(\mu+\alpha)$ and $n=\gamma+\mu+\omega>0$.

Local stability analysis of the disease-free equilibrium point of the System (4) presented in the following theorems.

\section{Theorem 3}

If $\Re_{0}<1$ and $m>0$, then the disease-free equilibrium point of the System (4), i.e. $E^{0}=$ $\left(\frac{K m}{r}, \frac{K \alpha m}{r\left(\mu+\gamma_{1}\right)}, 0\right)$ is locally asymptotically stable.

Proof.

The Jacobian matrix of the System (4) in $E^{0}$, i.e.

$$
J\left(E^{0}\right)=\left[\begin{array}{ccc}
-m & 0 & -\beta S^{0} \\
\alpha & -\left(\mu+\gamma_{1}\right) & -\beta_{1} V^{0} \\
0 & 0 & n\left(\Re_{0}-1\right)
\end{array}\right]
$$

Hence, the characteristics equation of $J\left(E^{0}\right)$, i.e.

$$
\left(\lambda-n\left(\Re_{0}-1\right)\right)(\lambda+m)\left(\lambda+\mu+\gamma_{1}\right)=0
$$

Then we have eigenvalue $\lambda_{1}=n\left(\Re_{0}-1\right), \lambda_{2}=-m$ dan $\lambda_{3}=-\mu-\gamma_{1}<0$. Therefore, if $\mathfrak{R}_{0}<1$ and $m>0$, then all the eigenvalues of $J\left(E^{0}\right)$ are negative consequently according to [19], $E^{0}$ is locally asymptotically stable.

Local stability analysis of the endemic equilibrium point of the System (4) presented in the following theorems.

\section{Theorem 4}

If $\mathfrak{R}_{0}>1, \beta_{1}>\beta$ and $m>0$, then the endemic equilibrium point of the System (4) denoted by $E_{1}^{*}=\left(S_{1}^{*}, V_{1}^{*}, I_{1}^{*}\right)$ is locally asymptotically stable with $S_{1}^{*}=\frac{K}{r}\left(m-\beta I^{*}\right), V_{1}^{*}=$ $\frac{K \alpha\left(m-\beta I^{*}\right)}{r\left(\beta_{1} I^{*}+\mu+\gamma_{1}\right)}$ and $I_{1}^{*}$ is the root of $g\left(I^{*}\right)=A_{1} I^{* 2}+A_{2} I+n\left(\mu+\gamma_{1}\right)\left(1-\mathfrak{R}_{0}\right), A_{1}=$ $\frac{K}{r} \beta^{2} \beta_{1}, A_{2}=\frac{K}{r} \beta^{2}\left(\mu+\gamma_{1}\right)+\frac{K}{r} \beta \beta_{1} \alpha+n \beta_{1}-\frac{K}{r} \beta \beta_{1} m$.

\section{Proof:}

The Jacobian matrix of the System (4) in $E_{1}^{*}$, i.e.

$$
J\left(E_{1}^{*}\right)=\left[\begin{array}{ccc}
-\frac{2 r}{K} S_{1}^{*}-\beta I_{1}^{*}+m & 0 & -\beta S_{1}^{*} \\
\alpha & -\beta_{1} I_{1}^{*}-\left(\mu+\gamma_{1}\right) & -\beta_{1} V_{1}^{*} \\
\beta I_{1}^{*} & \beta_{1} I_{1}^{*} & \beta S_{1}^{*}+\beta_{1} V_{1}^{*}-n
\end{array}\right]
$$

because $S_{1}^{*} \neq 0$ and according to Equation (5) we have

$r-\frac{r}{K} S_{1}^{*}-\beta I_{1}^{*}-(\mu+\alpha)=0 \Rightarrow m-\frac{2 r}{K} S_{1}^{*}-\beta I_{1}^{*}=-\frac{r}{K} S_{1}^{*}$ 
From Equation (6) we have

$$
\beta_{1} I_{1}^{*}+\mu+\gamma_{1}=\frac{\alpha S_{1}^{*}}{V_{1}^{*}}
$$

And clearly $\beta S_{1}^{*}+\beta_{1} V_{1}^{*}-n=0$. Hence, $J\left(E_{1}^{*}\right)$ equivalent with

$$
J\left(E_{1}^{*}\right)=\left[\begin{array}{ccc}
-\frac{r}{K} S_{1}^{*} & 0 & -\beta S_{1}^{*} \\
\alpha & -\frac{\alpha S_{1}^{*}}{V_{1}^{*}} & -\beta_{1} V_{1}^{*} \\
\beta I_{1}^{*} & \beta_{1} I_{1}^{*} & 0
\end{array}\right]
$$

Thus the characteristics equation of $J\left(E_{1}^{*}\right)$, i.e.

with

$$
a_{1} \lambda^{2}+a_{2} \lambda+a_{3}=0
$$

$a_{1}=\frac{\alpha S_{1}^{*}}{V_{1}^{*}}+\frac{r}{K} S_{1}^{*}$

$a_{2}=\frac{r \alpha S_{1}^{* 2}}{K V_{1}^{*}}+\beta^{2} S_{1}^{*} I_{1}^{*}+\beta_{1}^{2} V_{1}^{*} I_{1}^{*}$

$a_{3}=\beta \beta_{1} \alpha S_{1}^{*} I_{1}^{*}+\beta^{2} \alpha \frac{S_{1}^{* 2}}{V_{1}^{*}} I_{1}^{*}+\frac{r}{K} \beta_{1}^{2} S_{1}^{*} V_{1}^{*} I_{1}^{*}$

$a_{1} a_{2}-a_{3}=\frac{r \alpha^{2} S_{1}^{* 3}}{K V_{1}^{* 2}}+\frac{r^{2} \alpha S_{1}^{* 3}}{K^{2} V_{1}^{*}}+\frac{r \beta^{2}}{K} S_{1}^{* 2} I_{1}^{*}+\beta_{1} \alpha S_{1}^{*} I_{1}^{*}\left(\beta_{1}-\beta\right)$

Clearly $a_{1}>0, a_{2}>0, a_{3}>0$ and if $\beta_{1}>\beta$ then $a_{1} a_{2}-a_{3}>0$. Therefore, based on the Routh-Hurtwiz criteria if $\beta_{1}>\beta$, then all the eigenvalues of $J\left(E_{1}^{*}\right)$ are negative consequently the endemic equilibrium point of the System (4) denoted by $E_{1}^{*}$ Locally asymptotically stable.

\section{Numerical Simulations}

The following given numerical simulations to confirm the results obtained analytic related to local stability of disease-free equilibrium points and endemic to the System (1). Figure 1 is the local dynamics of the solution on the system (1) with the parameter value $K=1, r=0,04, \mu=0,01, \alpha=0,01, \beta=0,002, \beta_{1}=0,00045, \gamma=0,0001, \omega=$ $0,02, \gamma_{1}=0,004$ and initial conditions $S_{0}=0,8, V_{0}=0,1, I_{0}=0,1, R_{0}=0$. From the value of the parameter then we can calculate that $\mathfrak{R}_{0}=0,04<1, m=0,02>0$ and $E^{0}=$ $\left(S^{0}, V^{0}, I^{0}, R^{0}\right)=(0,5 ; 0,36 ; 0 ; 0,14)$. According to theorem 3 , the disease-free equilibrium point denoted by $E^{0}$ is locally asymptotically stable. These results correspond to the numerical simulation given. It means that the disease will disappear from the population.

Figure 2 is the local dynamics of the solution on the system (1) with the parameter value $K=1, r=0,04, \mu=0,01, \alpha=0,01, \beta=0,1, \beta_{1}=0,00045, \gamma=0,0001, \omega=$ $0,02, \gamma_{1}=0,004$ and initial conditions $S_{0}=0,8, V_{0}=0,1, I_{0}=0,1, R_{0}=0$. Clearly that $\beta_{1}>\beta$ then we can calculate that $\Re_{0}=1,66>1, m=0,02>0$ and the endemic equilibrium point denoted by $E_{1}^{*}=\left(S_{1}^{*}, V_{1}^{*}, I_{1}^{*}, R_{1}^{*}\right)$, i.e $E_{1}^{*}=(0,3 ; 0,21 ; 0,08 ; 0,09)$. According to theorem 3 , the endemic equilibrium point denoted by $E_{1}^{*}$ Locally asymptotically stable. These results correspond to the numerical simulation given. It means that the disease will remain in the population. 


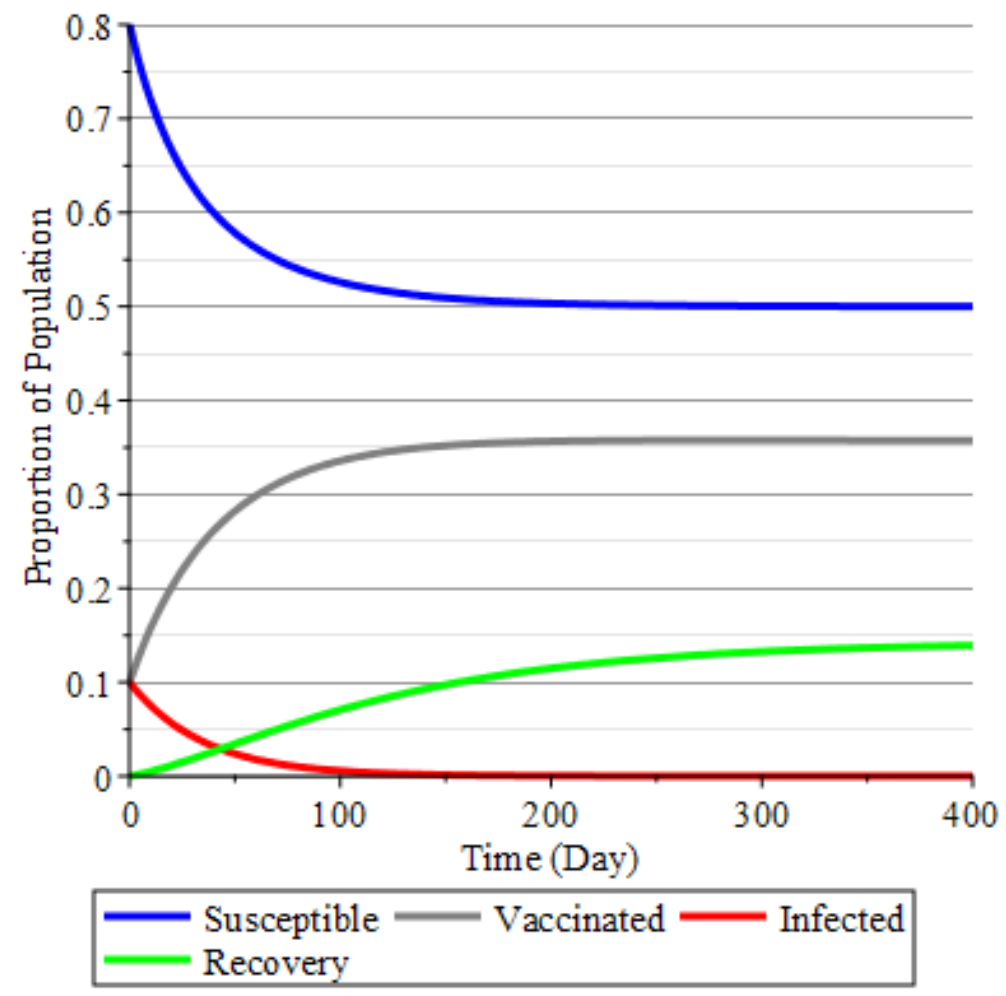

Figure 1. Dynamics of the solution on the System (1) for the case $\mathfrak{R}_{0}=0,04<1$

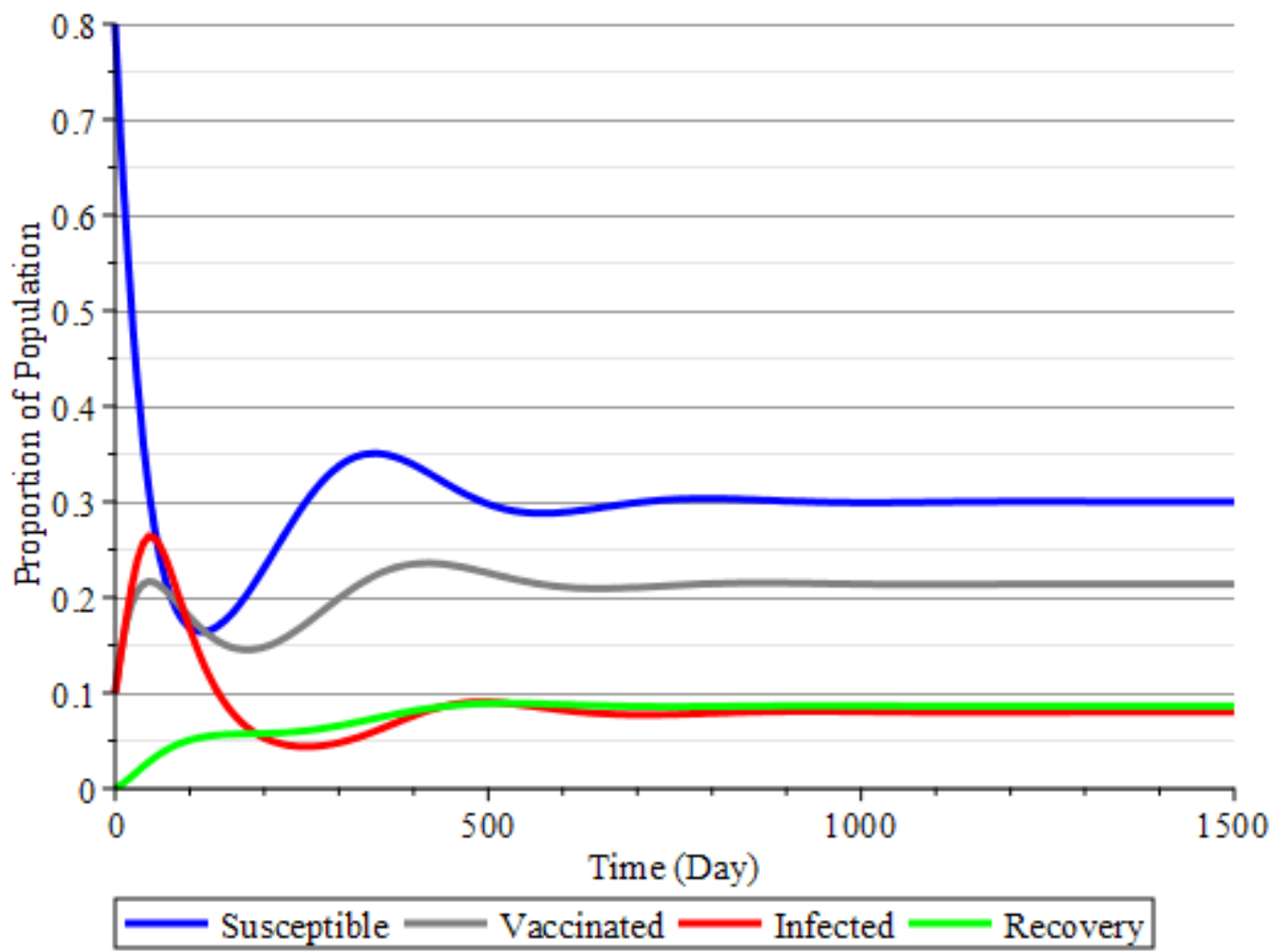

Figure 2. Dynamics of the solution on the System (1) for the case $\mathfrak{R}_{0}=1,66>1$ 


\section{Sensitivity Analysis of the Basic Reproduction Number}

The sensitivity analysis performed was a local sensitivity ([22], [23], and [24]). In this section, the parameters analyzed are $\beta, \alpha, \gamma_{1}, \gamma, \beta_{1}$. The sensitivity index for the parameter $\beta$ is

$$
C_{\beta}^{\Re_{0}}=\frac{\partial \Re_{0}}{\partial \beta} \times \frac{\beta}{\Re_{0}}=\frac{K \beta m\left(\mu+\gamma_{1}\right)}{K \beta m\left(\mu+\gamma_{1}\right)+\beta_{1} K \alpha m}
$$

With the same steps, we can obtain a sensitivity index for the other parameters. The following Table 1 is given the five parameter sensitivity to the basic reproduction number. The parameter $\beta, \alpha, \gamma_{1}, \gamma, \beta_{1}$ values used are $K=1, r=0,04, \mu=0,01, \alpha=0,01, \beta=$ $0,002, \beta_{1}=0,00045, \gamma=0,0001, \omega=0,02, \gamma_{1}=0,004$.

Table 1 . Sensitivity index of parameters to the basic reproduction number

\begin{tabular}{cc}
\hline Parameter & Sensitivity index \\
\hline$\beta$ & $+0,861$ \\
$\beta_{1}$ & +0.005 \\
$\alpha$ & $-0,138$ \\
$\gamma$ & $-0,001$ \\
$\gamma_{1}$ & -0.132 \\
\hline
\end{tabular}

Sensitivity index of parameter $\beta$ and $\beta_{1}$ has a positive value to $\Re_{0}$, it's mean that when the parameter $\beta$ and $\beta_{1}$ increases, then $\mathfrak{R}_{0}$ value also increases and conversely. The parameter sensitivity index of parameter $\alpha, \gamma, \gamma_{1}$ has a negative value to $\mathfrak{R}_{0}$, it's mean that when the parameter $\alpha, \gamma, \gamma_{1}$ increases, then $\Re_{0}$ value decreases, and conversely. The most influential parameters of the five parameters are transmission rate $(\beta)$ and vaccine rate $(\alpha)$.

\section{CONCLUSIONS}

This article is discussed the local stability analysis of the equilibrium point of the SVIR model adapted from Liu et al. The SVIR model discussed in this article was modified, assuming the growth rate on susceptible classes (V) approached with the growth of logistics. First, we determine the disease-free equilibrium point. Then, we find the basic reproduction number by using a disease-free equilibrium point that obtains. Next, we determine the existence of the endemic equilibrium point associated with the basic reproduction number $\left(\Re_{0}\right)$. Lastly, we determine the stability of the equilibrium point and illustrate the numerical simulation.

Our results show that if $\mathfrak{R}_{0}<1$ and $m>0$, then there exists a unique disease-free equilibrium point denoted by $E^{0}$ is locally asymptotically stable. The numerical simulation shown in Figure 1 supports these results. In the field of epidemiology, this means that the disease will disappear from the population. However, if $\mathfrak{R}_{0}>1, \beta_{1}>\beta$ and $m>0$, then the System (1) has two equilibrium point, i.e., the disease-free equilibrium point denoted by $E^{0}$ and the endemic equilibrium point denoted by $E_{1}^{*}$. In this case, the endemic equilibrium point denotes by $E_{1}^{*}$ Locally asymptotically stable. The numerical simulation shown in Figure 2 supports these results. In the field of epidemiology, this means that the disease will remain in the population. 


\section{REFERENCES}

[1] W. O. Kermack and A. G. McKendrick, "Contributions to the mathematical theory of epidemics-I,” Bull. Math. Biol., 1991.

[2] V. Capasso and G. Serio, "A generalization of the Kermack-McKendrick deterministic epidemic model," Math. Biosci., 1978.

[3] C. M. Kribs-Zaleta and J. X. Velasco-Hernández, "A simple vaccination model with multiple endemic states," Math. Biosci., 2000.

[4] J. Arino, C. C. Mccluskey, and P. V. Van Den Driessche, "Global results for an epidemic model with vaccination that exhibits backward bifurcation," SIAM J. Appl. Math., 2003.

[5] M. E. Alexander, C. Bowman, S. M. Moghadas, R. Summers, A. B. Gumel, and B. M. Sahai, "A vaccination model for transmission dynamics of influenza," SIAM J. Appl. Dyn. Syst., 2004.

[6] E. Shim, "A note on epidemic models with infective immigrants and vaccination," Mathematical Biosciences and Engineering. 2006.

[7] X. Liu, Y. Takeuchi, and S. Iwami, "SVIR epidemic models with vaccination strategies," J. Theor. Biol., 2008.

[8] M. A. Khan et al., "Stability analysis of an SVIR epidemic model with non-linear saturated incidence rate," Appl. Math. Sci., 2015.

[9] S. Islam, "Equilibriums and Stability of an SVIR Epidemic Model," BEST Int. J. Humanit. Arts, Med. Sci. (BEST IJHAMS) , 2015.

[10] J. Harianto, "Local Stability Analysis of an SVIR Epidemic Model," CAUCHY, 2017.

[11] J. Harianto and T. Suparwati, "SVIR Epidemic Model with Non Constant Population," CAUCHY, 2018.

[12] X. Z. Li, W. S. Li, and M. Ghosh, "Stability and bifurcation of an SIR epidemic model with nonlinear incidence and treatment," Appl. Math. Comput., 2009.

[13] J. J. Wang, J. Z. Zhang, and Z. Jin, "Analysis of an SIR model with bilinear incidence rate," Nonlinear Anal. Real World Appl., 2010.

[14] Q. Hu, Z. Hu, and F. Liao, "Stability and Hopf bifurcation in a HIV-1 infection model with delays and logistic growth," Math. Comput. Simul., 2016.

[15] Á. G. C. Pérez, E. Avila-Vales, and G. E. Garciá-Almeida, "Bifurcation analysis of an SIR model with logistic growth, nonlinear incidence, and saturated treatment," Complexity, 2019.

[16] E. Avila-Vales and Á. G. C. Pérez, "Dynamics of a time-delayed SIR epidemic model with logistic growth and saturated treatment," Chaos, Solitons and Fractals, 2019.

[17] X. Lai and X. Zou, "Modeling cell-to-cell spread of HIV-1 with logistic target cell growth," J. Math. Anal. Appl., 2015.

[18] R. Xu, Z. Wang, and F. Zhang, "Global stability and Hopf bifurcations of an SEIR epidemiological model with logistic growth and time delay," Appl. Math. Comput., 2015.

[19] L. Perko, Differential Equations and Dynamical Systems, 3th ed. New York: Springer, 2001.

[20] P. Van Den Driessche and J. Watmough, "Reproduction numbers and subthreshold endemic equilibria for compartmental models of disease transmission," Math. Biosci., 2002.

[21] P. van den Driessche, "Reproduction numbers of infectious disease models," Infect. Dis. Model., 2017.

[22] N. Chitnis, J. M. Hyman, and J. M. Cushing, "Determining important parameters in 
the spread of malaria through the sensitivity analysis of a mathematical model," Bull. Math. Biol., 2008.

[23] H. S. Rodrigues, M. T. T. Monteiro, and D. F. M. Torres, "Sensitivity Analysis in a Dengue Epidemiological Model," Conf. Pap. Math., 2013.

[24] P. J. Witbooi, G. E. Muller, and G. J. Van Schalkwyk, "Vaccination Control in a Stochastic SVIR Epidemic Model," Comput. Math. Methods Med., 2015. 